\title{
A replication study confirms the association of TNFSF4 (OX4OL) polymorphisms with systemic sclerosis in a large European cohort
}

Lara Bossini-Castillo, ${ }^{1}$ Jasper C A Broen, ${ }^{2}$ Carmen P Simeon, ${ }^{3}$ Lorenzo Beretta, ${ }^{4}$ Madelon C Vonk, ${ }^{2}$ Norberto Ortego-Centeno, ${ }^{5}$ Gerard Espinosa, ${ }^{6}$ Patricia Carreira, ${ }^{7}$ María Teresa Camps, ${ }^{8}$ Nuria Navarrete, ${ }^{9}$ María F González-Escribano, ${ }^{10}$ Esther VicenteRabaneda, ${ }^{11}$ Luis Rodríguez, ${ }^{12}$ Carlos Tolosa, ${ }^{13}$ José A Román-Ivorra, ${ }^{14}$ Inmaculada Gómez-Gracia, ${ }^{15}$ Francisco J García-Hernández, ${ }^{16}$ Iván Castellví, ${ }^{17}$ María Gallego, ${ }^{18}$ Antonio Fernández-Nebro, ${ }^{19}$ Rosa García-Portales, ${ }^{20}$ María Victoria Egurbide, ${ }^{21}$ Vicente Fonollosa, ${ }^{3}$ Paloma García de la Peña, ${ }^{22}$ Ana Pros, ${ }^{23}$ Miguel A González-Gay, ${ }^{24}$ Roger Hesselstrand, ${ }^{25}$ Gabriela Riemekasten, ${ }^{26}$ Torsten Witte, ${ }^{27}$ Marieke J H Coenen, ${ }^{28}$ Bobby P Koeleman, ${ }^{29}$ Frederic Houssiau, ${ }^{30}$ Vanessa Smith, ${ }^{31}$ Filip de Keyser, ${ }^{31}$ Rene Westhovens, ${ }^{32}$ Ellen De Langhe, ${ }^{32}$ Alexandre E Voskuyl, ${ }^{33}$ Annemie J Schuerwegh, ${ }^{34}$ Meng May Chee, ${ }^{35}$ Rajan Madhok, ${ }^{35}$ Paul Shiels, ${ }^{35}$ Carmen Fonseca, ${ }^{36}$ Christopher Denton, ${ }^{36}$ Kathleen Claes, ${ }^{37}$ Leonid Padykov, ${ }^{38}$ Annika Nordin, ${ }^{38}$ Øyvind Palm, ${ }^{39}$ Benedicte A Lie, ${ }^{40}$ Paolo Airó, ${ }^{41}$ Raffaella Scorza, ${ }^{4}$ Jacob M van Laar, ${ }^{42}$ Nicolas Hunzelmann, ${ }^{43}$ Alexander Kreuter, ${ }^{44}$ Ariane Herrick, ${ }^{45}$ Jane Worthington, ${ }^{45}$ Timothy R D J Radstake, ${ }^{2}$ Javier Martín, ${ }^{1}$ Blanca Rueda 1,10

\begin{abstract}
- Additional data
(supplementary tables 1-6 and supplementary figures 1-5) are published online only. To view these files please visit the journal online (http://ard. bmj.com).
\end{abstract}

For numbered affiliations see end of article

\section{Correspondence to Blanca Rueda, Instituto de Parasitología y Biomedicina López-Neyra, Consejo Superior de Investigaciones Científicas, Parque Tecnológico Ciencias de la Salud, Avenida del Conocimiento s/n, 18100 Armilla, Granada, Spain; blarume@ugr.es}

$\mathrm{JM}$ and $\mathrm{BR}$ contributed equally to this work.

Accepted 7 November 2010 Published Online First 27 December 2010

\section{ABSTRACT}

Objectives The aim of this study was to confirm the influence of TNFSF4 polymorphisms on systemic sclerosis (SSc) susceptibility and phenotypic features.

Methods A total of 8 European populations of Caucasian ancestry were included, comprising 3014 patients with SSc and 3125 healthy controls. Four genetic variants of TNFSF4 gene promoter (rs1234314, rs844644, rs844648 and rs12039904) were selected as genetic markers.

Results A pooled analysis revealed the association of rs1234314 and rs12039904 polymorphisms with SSc (OR $1.15,95 \% \mathrm{Cl} 1.02$ to 1.31 ; OR $1.18,95 \% \mathrm{Cl} 1.08$ to 1.29 , respectively). Significant association of the four tested variants with patients with limited cutaneous SSc (IcSSc) was revealed ( $\mathrm{r} 1234314 \mathrm{OR} 1.22,95 \% \mathrm{Cl} 1.07$ to 1.38; rs844644 OR 0.91, 95\% Cl 0.83 to 0.99; rs844648 OR $1.10,95 \% \mathrm{Cl} 1.01$ to 1.20 and rs12039904 OR 1.20, 95\% Cl 1.09 to 1.33). Association of rs 1234314 , rs 844648 and rs12039904 minor alleles with patients positive for anticentromere antibodies (ACA) remained significant (OR 1.23, $95 \% \mathrm{Cl} 1.10$ to 1.37 ; OR $1.12,95 \% \mathrm{Cl} 1.01$ to 1.25 ; OR $1.22,95 \%$ Cl 1.07 to 1.38 , respectively). Haplotype analysis confirmed a protective haplotype associated with SSc, IcSSc and ACA positive subgroups (OR 0.88, 95\% Cl 0.82 to 0.96 ; OR 0.88, 95\% $\mathrm{Cl} 0.80$ to 0.96 ; OR 0.86, 95\% $\mathrm{Cl} 0.77$ to 0.97 , respectively) and revealed a new risk haplotype associated with the same groups of patients (OR 1.14, 95\% Cl 1.03 to 1.26; OR 1.20, 95\% Cl 1.08 to 1.35; OR 1.23, 95\% Cl 1.07 to 1.42 , respectively).

Conclusions The data confirm the influence of TNFSF4 polymorphisms in SSc genetic susceptibility, especially in subsets of patients positive for IcSSc and ACA.

\section{INTRODUCTION}

Systemic sclerosis (SSc) is a connective tissue disorder characterised by fibrosis, vascular damage and immune imbalance. This pathology has a complex polygenic aetiology and variable clinical manifestations. Patients with SSc are commonly classified in two major subgroups: limited cutaneous systemic sclerosis (lcSSc) and diffuse cutaneous systemic sclerosis (dcSSc). ${ }^{1}$ Autoantibody status, especially anti-centromere antibodies (ACA) and anti-topoisomerase antibodies (ATA), is clinically used as prognostic biomarker. $^{1}$

Familial clustering and ethnic influences support the genetic component of this disease. ${ }^{2}$ Initially, only major histocompatibility complex (MHC) genes were firmly associated with SSc. ${ }^{2}$ Nevertheless, recently a number of candidate genes such as STAT4, BANK1 or IRF5, have been related to SSc genetic predisposition in independent populations by well powered studies. ${ }^{3}$ Hypothesis free approaches such as genome-wide association studies, have lately confirmed the role of MHC, IRF5 and STAT4 and uncovered new SSc susceptibility loci, such as CD247. ${ }^{5}$

In this line of research, four TNFSF4 promoter single nucleotide polymorphisms (SNPs) rs1234314, rs844644, rs844648 and rs12039904 were recently implicated in susceptibility to SSc in a Caucasian American population (composed of 1059 patients with SSc and 698 healthy controls). ${ }^{6}$ Interestingly, the TNFSF4 gene, which encodes OX40L, is considered as a potential autoimmunity candidate gene. OX40L is expressed on activated antigen presenting cells and endothelial cells in acute 
inflammation. Furthermore, it enhances B cell proliferation and differentiation, ${ }^{7}$ and its binding to $\mathrm{OX} 40$ (CD134) promotes proliferation and survival of T cells. ${ }^{8}$ All these processes could play an important role in loss of immune tolerance and pathology as observed in SSc.

On this basis, the aim of this study was to replicate the association of TNFSF4 gene promoter polymorphisms with SSc through a large association study in eight independent European populations of Caucasian ancestry, in order to confirm the implication of TNFSF4 gene in SSc genetic susceptibility and phenotypic features.

\section{PATIENTS AND METHODS \\ Patients}

A total of 3014 cases and 3125 controls from 8 European Caucasian cohorts (Spain, Germany, The Netherlands, Belgium, Italy, Sweden, Norway and UK) were included in this study. Patients with SSc were diagnosed accordingly with the 1980 American College of Rheumatology classification criteria for $\mathrm{SSc},{ }^{9}$ and were subdivided into those with $1 \mathrm{cSSc}$ and $\mathrm{dcSSc}$ as defined by LeRoy et al. ${ }^{10}$

The following clinical data was collected for ascertainment of clinical phenotype of patients with SSc: age, gender, disease duration and presence of SSc specific autoantibodies, ATA and ACA. Clinical subtype information was available for $82 \%$ of the patients, and autoantibody status was available for $74 \%$ of the patients. The control population consisted of unrelated healthy individuals recruited in the same geographical regions as patients with SSc, matched by age, sex and ethnicity. The local ethical committees at all participating centres approved the study. Patients and controls were included in the study after written informed consent was obtained.

\section{TNFSF4 polymorphisms genotyping}

SNPs rs1234314, rs844644, rs844648 and rs12039904 (tag-SNP of rs2205960 SNP) were genotyped using TaqMan SNP genotyping assays in a 7900HT Real-Time PCR System from Applied Biosystems (Foster City, California, USA). The genotyping call rate was over $93 \%$ in all cases and controls included.

\section{Statistical analysis}

Association was calculated by $2 \times 2$ contingency tables and Fisher's exact test or $\chi^{2}$ when necessary, obtaining $\mathrm{p}$ values, OR and 95\% CI using PLINK (V.1.06; http://pngu.mgh.harvard.edu/purcell/plink/). p Values below 0.05 after Benjamini and Hochberg False Discovery Rate Method correction were considered as statistically significant. Hardy-Weinberg equilibrium (HWE) was tested for all SNPs at significance level $=0.01$.

Haplotypes were constructed using PLINK (V.1.06) and Haploview V.4.2 (http://www.broadinstitute.org/haploview/ haploview). Haplotypes having a frequency $<5 \%$ in control groups were excluded for the analysis. Haplotype $p$ values were corrected using Bonferroni correction. Meta-analysis was carried out by PLINK (V.1.06) and StatsDirect (V.2.6.6; StatsDirect, Altrincham, UK) in the case of haplotypes. Homogeneity among cohorts was calculated using the Breslow-Day method, and OR calculation was performed under fixed effects model (Mantel-Haenszel) or random effects (DerSimonian-Laird) when necessary.

The power of the study for the whole set of patients and controls reached over 98\% (Power Calculator for Genetic Studies $\left.2006^{11}\right)$.

\section{RESULTS}

\section{Analysis of TNFSF4 promoter polymorphisms}

The allelic frequencies of the four SNPs tested were similar to those reported for Caucasian populations in previous studies 61213 and the international HapMap Project (http://hapmap.ncbi.nlm. nih.gov/). In addition, the genotypic distribution of healthy controls and SSc cases was in HWE for all SNPs.

Table 1 describes allelic distribution of the four SNPs in the pooled analysis, and supplementary tables 1-4 contain detailed data for each population. Pooled analysis of rs1234314 SNP showed statistically significant association of the $G$ allele with SSc ( $p=0.03$, OR $1.15,95 \%$ CI 1.02 to 1.31 ), with the subset of patients with $l_{c S S c}(\mathrm{p}=0.003$, OR $1.22,95 \%$ CI 1.07 to 1.38 ) and with patients positive for ACA ( $p=2.51 \mathrm{E}-04$, OR $1.23,95 \%$ CI 1.10 to 1.37 ) (table 1 and supplementary figure 1 ). The association of this genetic marker with $1 c S S c$ remained significant after the comparison of this subgroup of patients with those having $\mathrm{dcSSc}$ ( $\mathrm{p}=0.01$, OR $0.85,95 \%$ CI 0.75 to 0.96 , data not shown). Pooled analysis revealed a significant protective association of rs844644 minor allele with lcSSc ( $\mathrm{p}=0.03$, OR $0.91,95 \%$ CI 0.83 to 0.99 ) (table 1, supplementary figure 2). Similarly, the rs844648 A allele showed a significant association with susceptibility to lcSSc and ACA positive subgroups ( $p=0.04$, OR $1.10,95 \% \mathrm{CI}$ 1.01 to $1.20 ; \mathrm{p}=0.04$, OR $1.12,95 \%$ CI 1.01 to 1.25 , respectively) (table 1, supplementary figure 3 ). Pooled analysis revealed a strong association of rs12039904 T allele with patients with SSc ( $p=1.53 \mathrm{E}-04$, OR $1.18,95 \%$ CI 1.08 to 1.29$)$, with patients in the lcSSc subgroup ( $p=2.81 \mathrm{E}-04, \mathrm{OR} 1.20,95 \%$ CI 1.09 to 1.33 ) and patients in the ACA-positive subgroup ( $p=2.09 \mathrm{E}-03, \mathrm{OR} 1.22$, $95 \%$ CI 1.07 to 1.38 ) (table 1, supplementary figure 4).

\section{TNFSF4 haplotype analysis}

Haplotypes represented in over $5 \%$ of the healthy controls in any of the eight populations considered, were selected for pooled analysis (table 2 and supplementary table 5). Linkage disequilibrium patterns were tested in each of the eight cohorts analysed (supplementary table 6). Only two haplotypes reached significant association with SSc, CAGC ( $p=2.30 \mathrm{E}-03, \mathrm{OR} 0.88$, $95 \%$ CI 0.82 to 0.96 ) and GCAT ( $p=9.10 \mathrm{E}-03$, OR $1.14,95 \%$ CI 1.03 to 1.26 ) (supplementary table 5) (the order of the SNPs is rs1234314-rs844644-rs844648-rs12039904). Interestingly, the CAGC haplotype is composed by the protective alleles of all the tested SNPs while the GCAT haplotype harbours all the risk alleles. The association of CAGC and GCAT haplotypes with SSc clinical features remained significant for patients in the lcSSc ( $p=6.8 \mathrm{E}-03$, OR $0.88,95 \%$ CI 0.80 to 0.96 ; $p=1.3 \mathrm{E}-03$, OR $1.20,95 \%$ CI 1.08 to 1.35 , respectively, data not shown) and ACA-positive subsets ( $\mathrm{p}=0.01$, OR $0.86,95 \%$ CI 0.77 to 0.97 ; $\mathrm{p}=3.7 \mathrm{E}-03$, OR $1.23,95 \%$ CI 1.07 to 1.42 , respectively, data not shown).

\section{DISCUSSION}

TNFSF 4 polymorphisms have been related to susceptibility for different autoimmune diseases including SSc. ${ }^{6}{ }^{14}$ With the aim of validating the initially reported association of TNFSF 4 gene in SSc, we conducted a large case-control study and a pooled analysis in eight independent European populations of Caucasian ancestry.

In accordance with the report by Gourh et al, ${ }^{6}$ our study supports the implication of TNFSF4 gene promoter polymorphisms in SSc genetic predisposition. Stratification by SSc clinical subtype or autoantibody status confirmed the significant association of the TNFSF 4 variants with the patients in the lcSSc subset and 
ACA-positive subgroup but not with patients in the dcSSc or ATA-positive subsets. Nevertheless, the risk or protective directions in the associations were consistent with those reported by Gourh et al. ${ }^{6}$

Similarly, haplotype pooled analysis results obtained in the present study keep in with the findings from Gourh et al. ${ }^{6}$ The most represented haplotype in both reports are equivalent, appear in similar frequency and have a protective effect. Nevertheless, in our study the opposite haplotype GCAT, which could not be observed in the previous study, showed a significant risk association with SSc.

Interestingly, previous findings in systemic lupus erythematosus revealed the existence of equivalent protective and risk haplotypes to the ones reported in this study. Moreover, functional data showed that the risk haplotype produced an increased level of TNFSF 4 transcript (compared to the protective haplotype), and a higher surface expression of OX40L in lymphoblastoid cell lines and peripheral blood lymphocytes after activation. This overexpression seems to be related to the destruction of the DNA binding site for the transcriptional repressor E4BP4 (with a role in the survival of early B cell progenitors). ${ }^{12}$ Thus, the TNFSF4 risk haplotype associated with SSc and producing higher levels of OX40L might be implicated in the pathogenic mechanisms of SSc, by the alteration of regulatory processes controlling $\mathrm{B}$ and $\mathrm{T}$ cell proliferation and differentiation, leading to autoantibody production and tissue damage. ${ }^{7} 14-16$ Further

Table 1 Pooled analysis of TNFSF4 promoter genetic variants

\begin{tabular}{|c|c|c|c|c|c|c|}
\hline $\begin{array}{l}\text { SNP (minor/major alleles), } \\
\text { chromosome position (bp) }\end{array}$ & & $\mathbf{N}$ & MAF & $\mathbf{P}_{\mathrm{MH}}$ & $\mathbf{P}_{\mathrm{FDR}}$ & OR (95\% CI) \\
\hline \multirow[t]{6}{*}{ rs1234314 (G/C), 171444015} & Controls & 2920 & 0.41 & & & \\
\hline & SSc & 2856 & 0.44 & $0.03^{*} \dagger$ & - & 1.15 (1.02 to 1.31$)$ \\
\hline & IcSSc & 1608 & 0.46 & $0.003^{*} \ddagger$ & - & 1.22 (1.07 to 1.38$)$ \\
\hline & $\mathrm{dcSSc}$ & 724 & 0.42 & $0.75 \S$ & 0.84 & $1.02(0.91$ to 1.15$)$ \\
\hline & $\mathrm{ACA}+$ & 828 & 0.46 & $2.51 \mathrm{E}-04 \pi$ & 0.001 & 1.23 (1.10 to 1.37$)$ \\
\hline & ATA + & 519 & 0.42 & $0.23^{* *}$ & 0.43 & $1.08(0.95$ to 1.24$)$ \\
\hline \multirow[t]{6}{*}{ rs844644 (A/C), 171476118} & Controls & 2946 & 0.47 & & & \\
\hline & SSc & 2912 & 0.45 & $0.049 \dagger$ & 0.07 & $0.93(0.86$ to 1.00$)$ \\
\hline & IcSSc & 1653 & 0.45 & $0.03 \ddagger$ & 0.04 & 0.91 (0.83 to 0.99$)$ \\
\hline & dcSSc & 743 & 0.46 & $0.84 \S$ & 0.84 & $0.99(0.88$ to 1.11$)$ \\
\hline & $\mathrm{ACA}+$ & 856 & 0.44 & 0.0499 & 0.049 & $0.90(0.80$ to 1.00$)$ \\
\hline & ATA + & 533 & 0.46 & $0.33^{* *}$ & 0.43 & 0.94 (0.82 to 1.07$)$ \\
\hline \multirow[t]{6}{*}{ rs844648 (A/G), 171490486} & Controls & 2977 & 0.43 & & & \\
\hline & SSc & 2940 & 0.44 & $0.07 \dagger$ & 0.07 & $1.07(1.00$ to 1.15$)$ \\
\hline & IcSSc & 1673 & 0.45 & $0.04 \ddagger$ & 0.04 & $1.1(1.01$ to 1.20$)$ \\
\hline & dcSSc & 742 & 0.42 & $0.69 \S$ & 0.84 & $0.98(0.87$ to 1.10$)$ \\
\hline & $\mathrm{ACA}+$ & 860 & 0.45 & $0.04 \uparrow$ & 0.049 & $1.12(1.01$ to 1.25$)$ \\
\hline & ATA + & 529 & 0.42 & $0.74 * *$ & 0.74 & $1.02(0.89$ to 1.17$)$ \\
\hline \multirow[t]{6}{*}{ rs12039904 (T/C), 171478896} & Controls & 2991 & 0.23 & & & \\
\hline & SSc & 2894 & 0.26 & $1.53 \mathrm{E}-04 \dagger$ & $6.12 \mathrm{E}-04$ & 1.18 (1.08 to 1.29$)$ \\
\hline & IcSSc & 1639 & 0.26 & $2.81 \mathrm{E}-04 \ddagger$ & 5.61E-04 & $1.20(1.09$ to 1.33$)$ \\
\hline & dcSSc & 735 & 0.24 & $0.3 \S$ & 0.84 & $1.07(0.94$ to 1.23$)$ \\
\hline & $\mathrm{ACA}+$ & 840 & 0.26 & 2.09E-03ब & 4.17E-03 & 1.22 (1.07 to 1.38$)$ \\
\hline & ATA+ & 523 & 0.24 & $0.15^{* *}$ & 0.43 & $1.12(0.96$ to 1.31$)$ \\
\hline
\end{tabular}

Controls are used as reference for all comparisons.

*DerSimonian-Laird random effects model $p$ value.

†Breslow-Day rs1234314 $p=0.01 ;$ rs844644 $p=0.23$; rs844648 $p=0.52 ;$ rs $12039904 p=0.50$.

łBreslow-Day rs1234314 $p=0.08$; rs844644 $p=0.52 ;$ rs844648 $p=0.33 ;$ rs $12039904 p=0.48$.

$\S$ Breslow-Day rs1234314 $p=0.61 ;$ rs844644 $p=0.49 ;$ rs844648 $p=1.00 ;$ rs $12039904 p=0.38$

IBreslow-Day rs1234314 $p=0.29$; rs844644 $p=0.79$; rs844648 $p=0.94 ;$ rs12039904 $p=0.74$

**Beslow-Day rs1234314 $p=0.41 ;$ rs844644 $p=0.73$; rs844648 $p=0.71 ;$ rs12039904 $p=0.56$.

ACA, anti-centromere antibodies; ATA, anti-topoisomerase antibodies; chromosome position, position in chromosome 1; dcSSc, diffuse cutaneous systemic sclerosis; IcSSc, limited cutaneous systemic sclerosis; MAF, minor allele frequency; $\mathrm{P}_{\mathrm{FDR}}$, corrected $\mathrm{p}$ value using Benjamini and Hochberg False Discovery Rate; $\mathrm{P}_{\mathrm{MH}}$ allelic Mantel-Haenszel fixed effects model p value; SSc, systemic sclerosis.

Table 2 Pooled analysis of TNFSF4 haplotypes in patients with systemic sclerosis and controls

\begin{tabular}{|c|c|c|c|c|c|}
\hline (2n cases/2n controls) & Haplotype & Cases (\%) & Controls (\%) & $\mathbf{P}_{\mathrm{MH}}$ & OR (95\% CI) \\
\hline \multirow[t]{6}{*}{ Pooled (5222/5296) } & CAGC & 40.49 & 43.19 & $2.30 \mathrm{E}-03^{*}$ & $0.88(0.82$ to 0.96$)$ \\
\hline & CCGC & 10.23 & 10.04 & $0.47 \dagger \ddagger$ & $1.09(0.86$ to 1.39$)$ \\
\hline & GAGC & 4.43 & 3.75 & $0.08 \S$ & $1.20(0.98$ to 1.47$)$ \\
\hline & GCAC & 16.71 & 16.68 & $0.53 \pi$ & $0.97(0.87$ to 1.07$)$ \\
\hline & GCAT & 21.54 & 19.33 & $9.10 \mathrm{E}-03^{* *}$ & 1.14 (1.03 to 1.26$)$ \\
\hline & Others & 6.60 & 7.01 & $0.67^{*} \dagger \dagger$ & $0.94(0.73$ to 1.23$)$ \\
\hline
\end{tabular}

The order of the SNPs is rs1234314-rs844644-rs844648-rs12039904.

*Breslow-Day $\mathrm{p}=0.22$.

tDerSimonian-Laird random effects model $p$ value.

$\ddagger$ Breslow-Day $\mathrm{p}=0.004$.

$\S$ Breslow-Day $\mathrm{p}=0.62$

IBreslow-Day $\mathrm{p}=0.16$.

**Beslow-Day $p=0.24$.

††Breslow-Day $\mathrm{p}=0.01$.

$\mathrm{PMH}$, allelic Mantel-Haenszel fixed effects model $\mathrm{p}$ value. 
studies are necessary to elucidate the exact molecular mechanisms by which OX40L is implicated in SSc pathogenesis and more precisely how it can lead to the development of $1 \mathrm{cSSc}$ and ACA production.

In summary, our results confirm the implication of TNFSF4 promoter polymorphisms in SSc susceptibility, especially in patients in the 1cSSc and ACA-positive subgroups. These findings together with previous genetic and functional studies suggest TNFSF 4 as an interesting and consistent genetic factor for $\mathrm{SSc}$ and other autoimmune diseases and may open new opportunities for SSc treatment.

Acknowledgements We thank Sofía Vargas and Sonia Rodríguez for their excellent technical assistance, and all patients and donors for their collaboration. We are also thankful to EUSTAR (The EULAR Scleroderma Trials and Research group) for the facilitation of this project.

Funding This work was supported by grants SAF2009-11110, Junta de Andalucía, grants: CTS-4977 and CTS-180 and by RETICS Program, RD08/0075 (RIER) from Instituto de Salud Carlos III (ISCIII). BR was supported by ISCIII (Programa Sara Borrell). TRDJR was funded by the VIDI laureate from the Dutch association of research (NOW) and Dutch arthritis foundation (National Reumafonds). EDL is recipient of an Aspirant fellowship from FWO Vlaanderen (Flanders Research Foundation).

Ethical approval Ethical approval was obtained from the Ethics Committee in each hospital involved.

Provenance and peer review Not commissioned; externally peer reviewed.

Author affiliations ${ }^{1}$ Instituto de Parasitología y Biomedicina López-Neyra, CSIC, Granada, Spain

2Department of Rheumatology, Radboud University Nijmegen Medical Centre,

Nijmegen, The Netherlands

${ }^{3}$ Servicio de Medicina Interna, Hospital Valle de Hebron, Barcelona, Spain

${ }^{4}$ Referral Center for Systemic Autoimmune Diseases, University of Milan, Milan, Italy

${ }^{5}$ Servicio de Medicina Interna, Hospital Clínico Universitario, Granada, Spain

${ }^{6}$ Servicio de Medicina Interna, Hospital Clínico de Barcelona, Barcelona, Spain

${ }^{7}$ Servicio de Reumatología, Hospital 12 de Octubre, Madrid, Spain

${ }^{8}$ Servicio de Medicina Interna, Hospital Carlos-Haya, Málaga, Spain

${ }^{9}$ Servicio de Medicina Interna, Hospital Virgen de las Nieves, Granada, Spain

${ }^{10}$ Servicio de Inmunología, Hospital Virgen del Rocío, Sevilla, Spain

${ }^{11}$ Servicio de Reumatología, Hospital de la Princesa, Madrid, Spain

${ }^{12}$ Servicio de Reumatología, Hospital Clinico San Carlos, Madrid, Spain

${ }^{13}$ Servicio de Medicina Interna, Hospital Parc Tauli, Sabadell, Spain

${ }^{14}$ Servicio de Reumatología, Hospital del Doctor Peset aleixandre, Valencia, Spain

${ }^{15}$ Servicio de Reumatología, Hospital Reina Sofía, Córdoba, Spain

${ }^{16}$ Servicio de Medicina Interna, Hospital Virgen del Rocío, Sevilla, Spain

${ }^{17}$ Servicio de Reumatología, Hospital de Sant Pau, Barcelona, Spain

${ }^{18}$ Servicio de Medicina Interna, Hospital Central de Asturias, Oviedo, Spain

${ }^{19}$ Servicio de Reumatología, Hospital Carlos Haya, Málaga, Spain

${ }^{20}$ Servicio de Reumatología, Hospital Virgen de la Victoria, Málaga, Spain

${ }^{21}$ Servicio de Medicina Interna, Hospital de Cruces, Barakaldo, Spain

${ }^{22}$ Servicio de Reumatología, Hospital Ramón y Cajal, Madrid, Spain

${ }^{23}$ Servicio de Reumatología, Hospital Del Mar, Barcelona, Spain

${ }^{24}$ Servicio de Reumatología, Hospital Universitario Marqués de Valdecilla, Santander, Spain

${ }^{25}$ Department of Rheumatology, Lund University Hospital, Lund, Sweden

${ }^{26}$ Department of Rheumatology and Clinical Immunology, Charité University Hospital, Berlin, Germany

${ }^{27}$ Hannover Medical School, Hannover, Germany

${ }^{28}$ Department of Human Genetics, Radboud University Nijmegen Medical Centre,

Nijmegen, The Netherlands
${ }^{29}$ Section Complex Genetics, Department of Medical Genetics, University Medical

Center Utrecht, Utrecht, The Netherlands

30Université Catholique de Louvain (UCL), Brussels, Belgium

${ }^{31}$ University of Ghent, Ghent, Belgium

${ }^{32}$ University of Leuven (KULeuven), Leuven, Belgium

${ }^{33}$ Department of Rheumatology, VU University Medical Center, Amsterdam, The Netherlands

${ }^{34}$ Department of Rheumatology, Leiden University Medical Center, Leiden, The Netherlands

${ }^{35}$ University of Glasgow, Glasgow, UK

${ }^{36}$ Centre for Rheumatology, Royal Free and University College Medical School, London, UK

${ }^{37}$ Department of Genetics, University of Ghent, Ghent, Belgium

${ }^{38}$ Karolinska Institute, Stockholm, Sweden

${ }^{39}$ Department of Rheumatology, Rikshospitalet, Oslo University Hospital, Oslo, Norway

${ }^{40}$ Institute of Immunology, Rikshospitalet, Oslo University Hospital, Oslo, Norway

${ }^{41}$ Servizio di Reumatologia ed Immunologia Clinica Spedali Civili, Brescia, Italy

${ }^{42}$ Institute of Cellular Medicine, Newcastle University, Newcastle, UK

${ }^{43}$ Department of Dermatology, University of Cologne, Cologne, Germany

${ }^{44}$ Ruhr University of Bochum, Bochum, Germany

${ }^{45}$ Arthritis Research UK Epidemiology Unit, The University of Manchester, Manchester Academic Health Science Centre, Manchester, UK

\section{REFERENCES}

1. Steen VD. The many faces of scleroderma. Rheum Dis Clin North Am 2008;34:1-15; v.

2. Agarwal SK, Tan FK, Arnett FC. Genetics and genomic studies in scleroderma (systemic sclerosis). Rheum Dis Clin North Am 2008;34:17-40; v.

3. Agarwal SK, Reveille JD. The genetics of scleroderma (systemic sclerosis). Curr Opin Rheumatol 2010;22:133-8.

4. Zhou X, Lee JE, Arnett FC, et al. HLA-DPB1 and DPB2 are genetic loci for systemic sclerosis: a genome-wide association study in Koreans with replication in North Americans. Arthritis Rheum 2009;60:3807-14.

5. Radstake TR, Gorlova 0, Rueda B, et al. Genome-wide association study of systemic sclerosis identifies CD247 as a new susceptibility locus. Nat Genet 2010;42:426-9.

6. Gourh P, Arnett FC, Tan FK, et al. Association of TNFSF4 (OX40L) polymorphisms with susceptibility to systemic sclerosis. Ann Rheum Dis 2010;69:550-5.

7. Manku H, Graham DS, Vyse TJ. Association of the co-stimulator OX40L with systemic lupus erythematosus. J Mol Med 2009;87:229-34.

8. Gough MJ, Weinberg AD. OX40 (CD134) and OX40L. Adv Exp Med Biol 2009;647:94-107.

9. Preliminary criteria for the classification of systemic sclerosis (scleroderma). Subcommittee for scleroderma criteria of the American Rheumatism Association Diagnostic and Therapeutic Criteria Committee. Arthritis Rheum 1980;23:581-90.

10. LeRoy EC, Black C, Fleischmajer R, et al. Scleroderma (systemic sclerosis): classification, subsets and pathogenesis. J Rheumatol 1988;15:202-5.

11. Skol AD, Scott LJ, Abecasis GR, et al. Joint analysis is more efficient than replication-based analysis for two-stage genome-wide association studies. Nat Genet 2006;38:209-13.

12. Cunninghame Graham DS, Graham RR, Manku H, et al. Polymorphism at the TNF superfamily gene TNFSF4 confers susceptibility to systemic lupus erythematosus. Nat Genet 2008;40:83-9.

13. Delgado-Vega AM, Abelson AK, Sánchez E, et al. Replication of the TNFSF4 (OX40L) promoter region association with systemic lupus erythematosus. Genes Immun 2009;10:248-53.

14. Croft M. Control of immunity by the TNFR-related molecule OX40 (CD134). Annu Rev Immunol 2010;28:57-78.

15. Croft M, So T, Duan W, et al. The significance of OX40 and OX40L to T-cell biology and immune disease. Immunol Rev 2009;229:173-91.

16. Radstake TR, van Bon L, Broen J, et al. Increased frequency and compromised function of $\mathrm{T}$ regulatory cells in systemic sclerosis (SSC) is related to a diminished CD69 and TGFbeta expression. PLOS ONE 2009;4:e5981. 